\title{
A note on Lang's conjecture for quotients of bounded domains
}

\author{
Sébastien Boucksom and Simone Diverio \\ “... Voglio una vita che non è mai tardi, di quelle che non dormono mai...”
}

\begin{abstract}
It was conjectured by Lang that a complex projective manifold is Kobayashi hyperbolic if and only if it is of general type together with all of its subvarieties. We verify this conjecture for projective manifolds whose universal cover carries a bounded, strictly plurisubharmonic function. This includes in particular compact free quotients of bounded domains.
\end{abstract}

Keywords. Lang's conjecture, bounded domain, bounded strictly plurisubharmonic function, variety of general type, Bergman metric, $L^{2}$-estimates for $\bar{\partial}$-equation

2020 Mathematics Subject Classification. 32Q15; 32Q05

[Français]

Une note sur la conjecture de Lang pour les quotients des domaines bornés

Résumé. Lang a émis la conjecture qu'une variété projective complexe est Kobayashi hyperbolique si et seulement si elle est de type général ainsi que toutes ses sous-variétés. Nous vérifions cette conjecture pour les variétés projectives dont le revêtement universel admet une fonction strictement plurisousharmonique bornée. Ceci englobe en particulier les quotients compacts des domaines bornés par un groupe agissant librement.

Received by the Editors on January 24, 2020, and in final form on December 21, 2020.

Accepted on January 21, 2021.

Sébastien Boucksom

CNRS and CMLS, École Polytechnique, 91128 Palaiseau Cedex, France

e-mail: sebastien.boucksom@polytechnique.edu

Simone Diverio

Dipartimento di Matematica “Guido Castelnuovo”, SAPIENZA Università di Roma, Piazzale Aldo Moro 5, I-00185 Roma

e-mail: diverio@mat.uniromal.it

Both authors are partially supported by the ANR Programme: Défi de tous les savoirs (DS10) 2015, "GRACK", Project ID: ANR-15CE40-0003ANR. The second named author is also partially supported by the ANR Programme: Défi de tous les savoirs (DS10) 2016, "FOLIAGE", Project ID: ANR-16-CE40-0008

(C) by the author(s)

This work is licensed under http://creativecommons.org/licenses/by-sa/4.0/ 


\section{Contents}

1. Introduction. . . . . . . . . . . . . . . . . . . . . . . . . . . . . . . . 2

2. The Bergman metric and manifolds of general type . . . . . . . . . . . . . . . . . 4

3. Proof of Theorems $A$ and $B$. . . . . . . . . . . . . . . . . . . . . . . . . 5

4. Examples . . . . . . . . . . . . . . . . . . . . . . . . . . . . . . . . . 6

References. . . . . . . . . . . . . . . . . . . . . . . . . . 7

\section{Introduction}

For a compact complex space $X$, Kobayashi hyperbolicity is equivalent to the fact that every holomorphic map $\mathbb{C} \rightarrow X$ is constant, thanks to a classical result of Brody. When $X$ is moreover projective (or, more generally, compact Kähler), hyperbolicity is further expected to be completely characterized by (algebraic) positivity properties of $X$ and of its subvarieties. More precisely, we have the following conjecture, due to S. Lang.

Conjecture. [Lan86, Conjecture 5.6] A projective variety $X$ is hyperbolic if and only if every subvariety (including $X$ itself) is of general type.

Recall that a projective variety $X$ is of general type if the canonical bundle of any smooth projective birational model of $X$ is big, i.e. has maximal Kodaira dimension. This is for instance the case when $X$ is smooth and canonically polarized, i.e. with an ample canonical bundle $K_{X}$.

Note that Lang's conjecture in fact implies that every smooth hyperbolic projective manifold $X$ is canonically polarized, as conjectured in 1970 by S. Kobayashi. It is indeed a well-known consequence of the Minimal Model Program that any projective manifold of general type without rational curves is canonically polarized (see for instance [BBP13, Theorem A]).

Besides the trivial case of curves and partial results for surfaces [MM83, Des79, GG80, McQ98], Lang's conjecture is still almost completely open in higher dimension as of this writing. General projective hypersurfaces of high degree in projective space form a remarkable exception: they are known to be hyperbolic [Bro17] (see also [McQ99, DEG00, DT10, Siu04, Siu15, RY18]), and they satisfy Lang's conjecture [Cle86, Ein88, Xu94, Voi96, Pac04].

It is natural to test Lang's conjecture for the following two basic classes of manifolds, known to be hyperbolic since the very beginning of the theory:

(N) compact Kähler manifolds $X$ with negative holomorphic sectional curvature;

(B) compact, free quotients $X$ of bounded domains $\Omega \Subset \mathbb{C}^{n}$.

In case $(\mathrm{N})$, ampleness of $K_{X}$ was established in [WY16a, WY16b, TY17] (see also [DT19]). By curvature monotonicity, this implies that every smooth subvariety of $X$ also has ample canonical bundle. More generally, Guenancia recently showed [Gue18] that each (possibly singular) subvariety of $X$ is of general type, thereby verifying Lang's conjecture in that case. One might even more generally consider the case where $X$ carries an arbitrary Hermitian metric of negative holomorphic sectional curvature, which seems to be still open.

In this note, we confirm Lang's conjecture in case (B). While the case of quotients of bounded symmetric domains has been widely studied (see, just to cite a few, [Nad89, BKT13, Bru16, Cad16, Rou16, RT18]), the 
general case seems to have somehow passed unnoticed. Instead of bounded domains, we consider more generally the following class of manifolds, which comprises relatively compact domains in Stein manifolds, and has the virtue of being stable under passing to an étale cover or a submanifold.

Definition. We say that a complex manifold $M$ is of bounded type if it carries a bounded, strictly plurisubharmonic $^{1}$ function $\varphi$.

By a well-known result of Richberg, any continuous bounded strictly psh function on a complex manifold $M$ can be written as a decreasing limit of smooth strictly psh functions, but this fails in general for discontinuous functions [FS87, p. 66], and it is thus unclear to us whether every manifold of bounded type should carry also a smooth bounded strictly psh function.

Theorem A. Let $X$ be a compact Kähler manifold admitting an étale (Galois) cover $\tilde{X} \rightarrow X$ with $\tilde{X}$ of bounded type. Then:

(i) X is Kobayashi hyperbolic;

(ii) X has large fundamental group;

(iii) $X$ is projective and canonically polarized;

(iv) every subvariety of $X$ is of general type.

Note that $\tilde{X}$ can always be replaced with the universal cover of $X$, and hence can be assumed to be Galois. Manifolds to which the above theorem applies include compact free quotients of bounded symmetric domains, and Kodaira surfaces (see $§ 4$ for more details).

By [Kob98, Theorem 3.2.8], (i) in Theorem A holds iff $\tilde{X}$ is hyperbolic, which follows from the fact that manifolds of bounded type are Kobayashi hyperbolic [Sib81, Theorem 3] (alternatively, any entire curve $f: \mathbb{C} \rightarrow X$ lifts to $\tilde{X}$, and the pull-back to $\mathbb{C}$ of the bounded, strictly psh function carried by $\tilde{X}$ has to be constant, showing that $f$ itself is constant).

By definition, (ii) means that the image in $\pi_{1}(X)$ of the fundamental group of any subvariety $Z \subseteq X$ is infinite [Kol95, §4.1], and is a direct consequence of the fact that manifolds of bounded type do not contain nontrivial compact subvarieties. According to the Shafarevich conjecture, $\tilde{X}$ should in fact be Stein; in case $\tilde{X}$ is a bounded domain of $\mathbb{C}^{n}$, this is indeed a classical result of Siegel [Sie50] (see also [Kob59, Theorem 6.2]).

By another classical result, this time due to Kodaira [Kod54], any compact complex manifold $X$ admitting a Galois étale cover $\tilde{X} \rightarrow X$ biholomorphic to a bounded domain in $\mathbb{C}^{n}$ is projective, with $K_{X}$ ample. Indeed, the Bergman metric of $\tilde{X}$ is non-degenerate, and it descends to a positively curved metric on $K_{X}$. Our proof of (iii) and (iv) is a simple variant of this idea, inspired by [CZ02]. For each subvariety $Y \subseteq X$ with desingularization $Z \rightarrow Y$ and induced Galois étale cover $\tilde{Z} \rightarrow Z$, we use basic Hörmander-AndreottiVesentini-Demailly $L^{2}$-estimates for $\bar{\partial}$ to show that the Bergman metric of $\tilde{Z}$ is generically non-degenerate. It then descends to a psh metric on $K_{Z}$, smooth and strictly psh on a nonempty Zariski open set, which is enough to conclude that $K_{Z}$ is big, by [Bou02].

The above reasoning actually yields the following variant of Theorem A, which is perhaps worth mentioning.

Theorem B. Let X be a compact Kähler manifold whose universal cover carries a bounded psh function which is strictly psh at one point. Then:

(i) the manifold $X$ is of general type (and hence projective), and there exists a closed proper subset $\Sigma \subset X$ (in the Euclidean topology) such that

(ii) any subvariety of $X$ not contained in $\sum$ is of general type;

\footnotetext{
${ }^{1}$ i.e. each point of $M$ admits a coordinate neighbourhood on which $\varphi-c|z|^{2}$ is psh for some $c>0$.
} 
(iii) the manifold $X$ is Kobayashi hyperbolic modulo $\Sigma$; in particular, every entire curve $\mathbb{C} \rightarrow X$ is contained in $\Sigma$.

A result very similar to (i) of Theorem B appears in [Che03, Proposition 2.3], where the psh function is further assumed to be smooth. See also [Kik11, Kik13], especially [Kik13, Lemma 3.4], for other related results.

Unfortunately, at the moment we are not able to show that the proper subset $\Sigma$ in the statement above can be chosen to be Zariski closed. If it were the case, this would confirm the (strong) Green-Griffiths-Lang conjecture for this class of projective manifolds of general type.

As a final comment, note that Kähler hyperbolic manifolds in the sense of Gromov, i.e. compact Kähler manifolds $X$ carrying a Kähler metric $\omega$ whose pull-back to the universal cover $\pi: \tilde{X} \rightarrow X$ satisfies $\pi^{*} \omega=d \alpha$ with $\alpha$ bounded, also satisfy (i)-(iii) in Theorem A [Gro91]. It would be interesting to check Lang's conjecture for such manifolds as well.

Acknowledgment. This work was started during the first-named author's stay at SAPIENZA Università di Roma. He is very grateful to the mathematics department for its hospitality, and to INdAM for financial support.

The second-named author warmly thanks Laura Geatti for indicating the reference [Han57].

Both authors would also like to thank Stefano Trapani for helpful discussions, in particular for pointing out the reference [FS87], as well as the anonymous referee for several useful observations and remarks, in particular for suggesting to add the statement which corresponds to Theorem B.

\section{The Bergman metric and manifolds of general type}

We start by recalling that the Bergman space of a complex manifold $M$ is the separable Hibert space $\mathcal{H}=\mathcal{H}(M)$ of holomorphic forms $\eta \in H^{0}\left(M, K_{M}\right)$ such that

$$
\|\eta\|_{\mathcal{H}}^{2}:=i^{n^{2}} \int_{\tilde{X}} \eta \wedge \bar{\eta}<\infty
$$

with $n=\operatorname{dim} M$. Assuming $\mathcal{H} \neq\{0\}$, we get an induced (possibly singular) psh metric $h_{M}$ on $K_{M}$, invariant under $\operatorname{Aut}(M)$, characterized pointwise by

$$
h / h_{M}=\sup _{\eta \in \mathcal{H} \backslash\{0\}} \frac{|\eta|_{h}^{2}}{\|\eta\|_{\mathcal{H}}^{2}}=\sum_{j}\left|\eta_{j}\right|_{h}^{2},
$$

for any choice of smooth metric $h$ on $K_{M}$ and orthonormal basis $\left(\eta_{j}\right)$ for $\mathcal{H}$ (see for instance [Kob98, §4.10]).

The curvature current of $h_{M}$ is classically called the "Bergman metric" of $M$; it is a bona fide Kähler form precisely on the Zariski open subset of $M$ consisting of points at which $\mathcal{H}$ generates 1 -jets [Kob98, Proposition 4.10.11].

Definition 2.1. We shall say that a complex manifold $M$ has a non-degenerate (resp. generically non-degenerate) Bergman metric if its Bergman space $\mathcal{H}$ generates 1 -jets at each (resp. some) point of $M$.

We next recall the following standard consequence of $L^{2}$-estimates for $\bar{\partial}$.

Lemma 2.2. Let $M$ be a complete Kähler manifold with a bounded psh function $\varphi$. If $\varphi$ is strictly psh on $M$ (resp. at some point of $M$ ), then the Bergman metric of $M$ is non-degenerate (resp. generically non-degenerate).

Proof. Pick a complete Kähler metric $\omega$ on $M$. Assume $\varphi$ strictly psh at $p \in M$, and fix a coordinate ball $(U, z)$ centered at $p$ with $\varphi$ strictly psh near $\bar{U}$. Pick also $\chi \in C_{c}^{\infty}(U)$ with $\chi \equiv 1$ near $p$. Since $\chi \log |z|$ is 
strictly psh in an open neighbourhood $V$ of $p$, smooth on $U \backslash \bar{V}$, and compactly supported in $U$, we can then choose $A \gg 1$ such that

$$
\psi:=(n+1) \chi \log |z|+A \varphi
$$

is psh on $M$, with $d d^{c} \psi \geq \omega$ on $U$. Note that $\psi$ is also bounded above on $M, \varphi$ being assumed to be bounded.

For an appropriate choice of holomorphic function $f$ on $U$, the smooth $(n, 0)$-form $\eta:=\chi f d z_{1} \wedge \cdots \wedge d z_{n}$, which is compactly supported in $U$ and holomorphic in a neighborhood of $x$, will have any prescribed jet at $p$. The $(n, 1)$-form $\bar{\partial} \eta$ is compactly supported in $U$, and identically zero in a neighborhood of $p$, so that $|\bar{\partial} \eta|_{\omega} e^{-\psi} \in L^{2}(U)$. Since $d d^{c} \psi \geq \omega$ on $U$, [Dem82, Théorème 5.1] yields an $L_{\text {loc }}^{2}(n, 0)$-form $u$ on $M$ such that $\bar{\partial} u=\bar{\partial} \eta$ and

$$
i^{n^{2}} \int_{M} u \wedge \bar{u} e^{-2 \psi} \leq \int_{U}|\bar{\partial} \eta|_{\omega}^{2} e^{-2 \psi} d V_{\omega} .
$$

As a result, $v:=\eta-u$ is a holomorphic $n$-form on $X$. Since $u=\eta-v$ is holomorphic at $x$ and $\psi$ has an isolated singularity of type $(n+1) \log |z|$ at $x,(2.1)$ forces $u$ to vanish to order 2 at $p$, so that $v$ and $\eta$ have the same 1 -jet at $p$. Finally, $(2.1)$ and the fact that $\psi$ is bounded above on $M$ shows that $u$ is $L^{2}$. Since $\eta$ is clearly $L^{2}$ as well, $v$ belongs to the Bergman space $\mathcal{H}$, with given jet at $p$, and we are done.

Suppose now that $X$ is a compact complex manifold, and $\tilde{X} \rightarrow X$ is a Galois étale cover. Assume that the Bergman metric of $\tilde{X}$ is non-degenerate, so that the canonical metric $h_{\tilde{X}}$ on $K_{\tilde{X}}$ defined by $\mathcal{H}(\tilde{X})$ is smooth, strictly psh. Being invariant under automorphisms, this metric descends to a smooth, strictly psh metric on $K_{X}$, and the latter is thus ample by [Kod54]. This argument, which goes back to the same paper by Kodaira, admits the following variant.

Lemma 2.3. Let $X$ be a compact Kähler manifold admitting a Galois étale cover $\tilde{X} \rightarrow X$ with generically non-degenerate Bergman metric. Then $X$ is projective and of general type.

Proof. The assumption now means that the psh metric $h_{\tilde{X}}$ on $K_{\tilde{X}}$ is smooth and strictly psh on a non-empty Zariski open subset. It descends again to a psh metric on $K_{X}$, smooth and strictly psh on a non-empty Zariski open subset, and we conclude that $K_{X}$ is big by [Bou02, §2.3] (see also [BEGZ10, §1.5]). Being both Moishezon and Kähler, $X$ is then projective.

Remark 2.4. Combining Lemma 2.2 with Lemma 2.3 one thus obtains that a compact Kähler manifold $X$ admitting a Galois étale cover $\tilde{X}$ which supports a bounded psh function that is strictly psh at some point is projective and of general type. It is perhaps worth noticing that a proof of this statement can also be obtained replacing the use of the first-named author's criterion in Lemma 2.3 by more elementary arguments using Poincaré series, following Gromov [Gro91, Corollary 3.2.B] (see also [Kol95, Chapter 13] for a very nice account of the method of Gromov). Indeed, the fact that the Galois cover $\tilde{X}$ posseses a bounded psh function that is strictly psh at some point implies both that $X$ has generically large fundamental group and that $K_{\tilde{X}}$ has a non-zero holomorphic $L^{2}$ section, the latter thanks to Lemma 2.2. At this point one can use directly [Kol95, 13.10 Corollary].

\section{Proof of Theorems A and B}

We finally prove the theorems stated in the introduction.

\subsection{Proof of Theorem A}

Let $X$ be a compact Kähler manifold with an étale cover $\pi: \tilde{X} \rightarrow X$ of bounded type, which may be assumed to be Galois after replacing $\tilde{X}$ by the universal cover of $X$. Since $\tilde{X}$ is also complete Kähler, its 
Bergman metric is non-degenerate by Lemma 2.2, and $X$ is thus projective and canonically polarized by [Kod54].

Now let $Y \subseteq X$ be an irreducible subvariety. On the one hand, pick any connected component $\tilde{Y}$ of the preimage $\pi^{-1}(Y) \subset \tilde{X}$, so that $\pi$ induces a Galois étale cover $\left.\pi\right|_{\tilde{Y}}: \tilde{Y} \rightarrow Y$. On the other hand, let $\mu: Z \rightarrow Y$ be a projective modification with $Z$ smooth and $\mu$ isomorphic over $Y_{\text {reg, }}$, whose existence is guaranteed by Hironaka. Since $Y$ is Kähler and $\mu$ is projective, $Z$ is then a compact Kähler manifold. The fiber product $\tilde{Z}=Z \times_{Y} \tilde{Y}$ sits in the following diagram

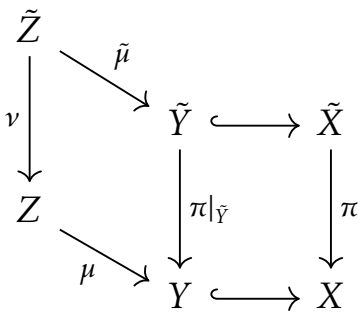

Being a base change of a Galois étale cover, $v$ is a Galois étale cover, and $\tilde{\mu}$ is a resolution of singularities of

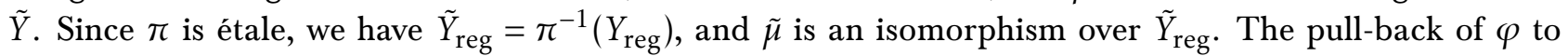
$\tilde{Z}$ is thus a bounded psh function, strictly psh at any point $p \in \tilde{\mu}^{-1}\left(\tilde{Y}_{\text {reg }}\right)$. Since $Z$ is compact Kähler, $\tilde{Z}$ is complete Kähler. By Lemma 2.2, the Bergman metric of $Z$ is generically non-degenerate, and $Z$ is thus of general type, by Lemma 2.3 .

\subsection{Proof of Theorem B}

The first point of the statement follows directly from Lemma 2.2 combined with Lemma 2.3.

Next, let $\tilde{\Sigma} \subset \tilde{X}$ be the set of points at which no bounded psh function on $\tilde{X}$ is strictly psh. This set is closed in the Euclidean topology and clearly invariant under holomorphic automorphisms of $\tilde{X}$. In particular, its image $\Sigma \subset X$ under the covering map is a proper closed subset.

For point (ii), the proof proceeds exactly as for the proof of point (iv) of Theorem A above. Indeed, we only used the following: given a subvariety $Y \subseteq X$ there exists a point on the regular locus of $Y$ whose preimage in the universal cover of $X$ contains a point of strict plurisubharmonicity of a bounded psh function on $\tilde{X}$. In our more general situation here, this happens precisely when $Y$ is not entirely contained in $\Sigma$.

Finally, for point (iii), by [Sib81, Theorem 3] we have that $\tilde{X}$ is hyperbolic at every point outside $\tilde{\Sigma}$. By this we mean that the infinitesimal Kobayashi metric of $\tilde{X}$ is locally uniformly bounded below away from zero at every such point. Therefore, the Kobayashi (pseudo)distance - which is the integrated form of the infinitesimal Kobayashi metric - of any two distinct points of $\tilde{X}$ must be positive unless they both belong to $\tilde{\Sigma}$, which is precisely the definition of $\tilde{X}$ being hyperbolic modulo $\tilde{\Sigma}$. Since $\tilde{\Sigma}$ is invariant and closed, by [Kob98, Theorem 3.2.32] we get that $X$ is hyperbolic modulo $\sum$.

In particular, if $f: \mathbb{C} \rightarrow X$ is any holomorphic map, then any two distinct points in the image of $f$ have zero Kobayashi distance and must therefore sit inside $\Sigma$.

\section{Examples}

The goal of this section is to briefly discuss examples of manifolds of bounded type with a compact free quotient. As we shall see, the list is unfortunately quite short.

Consider first a bounded domain $\Omega \Subset \mathbb{C}^{n}$ that admits a compact free quotient.

- By [Sie50], $\Omega$ is automatically pseudoconvex (see also [Kob59, Theorem 6.2]).

- If $\partial \Omega$ is $C^{2}$, then $\Omega$ is biholomorphic to a ball [Won77, Ros79] (this already holds when $\partial \Omega$ is $C^{1,1}$, by a recent result of Zimmer [Zim19]). 
- If $\Omega$ is homogeneous, then the Lie group $\operatorname{Aut}(\Omega)$ admits a (uniform) lattice, hence is unimodular, and $\Omega$ is thus necessarily symmetric [Han57, Theorem IV].

- If $\Omega$ is convex hyperbolic, or if $\Omega$ is irreducible and Aut $(\Omega)$ is positive dimensional, then $\Omega$ is symmetric [Fra89, Fra95].

Bounded symmetric domains. - As mentioned in the introduction, a classical result of A. Borel conversely implies that any bounded symmetric domain admits a compact free quotient [Bor63].

But even in this extensively studied framework, as far as we know, the first proof of the fact that compact free quotients of bounded symmetric domains satisfy Lang's conjecture (which corresponds to part (iv) of our Theorem A) is quite recent [BKT13]. The proof by Brunebarbe-Klingler-Totaro is anyway of a different nature and appears to be perhaps less direct than ours, concerning mostly the negativity properties of the (holomorphic sectional and bisectional) curvature of the Bergman metric, which are under control in the symmetric case only.

In this spirit, the same result also follows from Guenancia's work [Gue18], since bounded symmetric domains enter the (a priori much larger) class (N) mentioned in the introduction (observe that while in [BKT13] both the holomorphic bisectional and sectional curvatures have to be under control, [Gue18] only requires a negative bound from above for the holomorphic sectional curvature).

Universal covers of Kodaira fibrations. - Examples of non-symmetric (and hence non-homogeneous) bounded domains with a compact free quotient arise from universal covers of Kodaira fibrations.

Recall that the latter are non-isotrivial holomorphic submersions with connected fibers $f: S \rightarrow C$ from a smooth projective surface to a smooth curve. Both the fiber and the base are then of genus at least 2, examples arising for instance from the (projective) Satake compactification of the moduli space of curves of genus $g \geq 3$ (see [BHPV04, §V.14]).

By [Gri71], the universal cover $\tilde{S}$ is biholomorphic to a bounded domain $\Omega \Subset \mathbb{C}^{2}$, which is not symmetric.

To see this, it is enough to check that $c_{1}^{2}(S) \neq 3 c_{2}(S)$ and $c_{1}(S)^{2} \neq 2 c_{2}(S)$, by the Hirzebruch proportionality principle (since the only bounded symmetric domains in $\mathbb{C}^{2}$ are the ball and the bi-disc). While it is

straightforward to see that the topological index $\tau(S)=\frac{1}{3}\left(c_{1}^{2}(S)-2 c_{2}(S)\right)$ of a Kodaira fibration is always strictly positive, $c_{1}(S)^{2} \neq 3 c_{2}(S)$ is more involved [Liu96].

Observe that for this family of examples, verifying Lang's conjecture is however straightforward since Kodaira fibrations are classically known to be of general type and, being Kobayashi hyperbolic surfaces, they cannot contain any (possibly singular) rational or elliptic curve.

\section{References}

[BHPV04] W. P. Barth, K. Hulek, C. A. M. Peters and A. Van de Ven, Compact complex surfaces, second ed., Ergebnisse der Mathematik und ihrer Grenzgebiete. 3. Folge. A Series of Modern Surveys in Mathematics [Results in Mathematics and Related Areas. 3rd Series. A Series of Modern Surveys in Mathematics], vol. 4, Springer-Verlag, Berlin, 2004.

[Bor63] A. Borel, Compact Clifford-Klein forms of symmetric spaces, Topology 2 (1963), 111-122.

[Bou02] S. Boucksom, On the volume of a line bundle, Internat. J. Math. 13 (2002), no. 10, 1043-1063.

[BBP13] S. Boucksom, A. Broustet and G. Pacienza, Uniruledness of stable base loci of adjoint linear systems via Mori theory, Math. Z. 275 (2013), no. 1-2, 499-507.

[BEGZ10] S. Boucksom, P. Eyssidieux, V. Guedj and A. Zeriahi, Monge-Ampère equations in big cohomology classes, Acta Math. 205 (2010), no. 2, 199-262. 
[Brol7] D. Brotbek, On the hyperbolicity of general hypersurfaces, Publ. Math. Inst. Hautes Études Sci. 126 (2017), 1-34.

[Bru16] Y. Brunebarbe, A strong hyperbolicity property of locally symmetric varieties, preprint arXiv:1606.03972 (2016), to appear in Ann. Sci. Éc. Norm. Supér.

[BKT13] Y. Brunebarbe, B. Klingler and B. Totaro, Symmetric differentials and the fundamental group, Duke Math. J. 162 (2013), no. 14, 2797-2813.

[Cad16] B. Cadorel, Symmetric differentials on complex hyperbolic manifolds with cusps, preprint arXiv:1606.05470 (2016), to appear in J. Differential Geom.

[Che03] B.-Y. Chen, The Bergman metric on complete Kähler manifolds, Math. Ann. 327 (2003), no. 2, 339-349.

[CZ02] B.-Y. Chen and J.-H. Zhang, The Bergman metric on a Stein manifold with a bounded plurisubharmonic function, Trans. Amer. Math. Soc. 354 (2002), no. 8, 2997-3009.

[Cle86] H. Clemens, Curves on generic hypersurfaces, Ann. Sci. École Norm. Sup. (4) 19 (1986), no. 4, 629-636.

[Dem82] J.-P. Demailly, Estimations $L^{2}$ pour l'opérateur $\partial ̄$ d'un fibré vectoriel holomorphe semi-positif au-dessus d'une variété kählérienne complète, Ann. Sci. École Norm. Sup. (4) 15 (1982), no. 3, 457-511.

[DEG00] J.-P. Demailly and J. El Goul, Hyperbolicity of generic surfaces of high degree in projective 3-space, Amer. J. Math. 122 (2000), no. 3, 515-546.

[Des79] M. Deschamps, Courbes de genre géométrique borné sur une surface de type général [d'après $F$. A. Bogomolov], Séminaire Bourbaki, 30e année (1977/78), Lecture Notes in Math., vol. 710, Springer, Berlin, 1979, Exp. No. 519, pp. 233-247.

[DT10] S. Diverio and S. Trapani, A remark on the codimension of the Green-Griffiths locus of generic projective hypersurfaces of high degree, J. Reine Angew. Math. 649 (2010), 55-61.

[DT19] - Quasi-negative holomorphic sectional curvature and positivity of the canonical bundle, J. Differential Geom. 111 (2019), no. 2, 303-314.

[Ein88] L. Ein, Subvarieties of generic complete intersections, Invent. Math. 94 (1988), no. 1, 163-169.

[FS87] J. E. Fornæss and B. Stensønes, Lectures on counterexamples in several complex variables, Mathematical Notes, vol. 33, Princeton University Press, Princeton, NJ; University of Tokyo Press, Tokyo, 1987.

[Fra89] S. Frankel, Complex geometry of convex domains that cover varieties, Acta Math. 163 (1989), no. 1-2, 109-149.

[Fra95] L Locally symmetric and rigid factors for complex manifolds via harmonic maps, Ann. of Math. (2) 141 (1995), no. 2, 285-300.

[GG80] M. Green and P. A. Griffiths, Two applications of algebraic geometry to entire holomorphic mappings, The Chern Symposium 1979 (Proc. Internat. Sympos., Berkeley, Calif., 1979), Springer, New York-Berlin, 1980, pp. 41-74.

[Gri71] P. A. Griffiths, Complex-analytic properties of certain Zariski open sets on algebraic varieties, Ann. of Math. (2) 94 (1971), 21-51.

[Gro91] M. Gromov, Kähler hyperbolicity and L L $_{2}$-Hodge theory, J. Differential Geom. 33 (1991), no. 1, 263-292.

[Gue18] H. Guenancia, Quasi-projective manifolds with negative holomorphic sectional curvature, preprint arXiv:1808.01854 (2018), to appear in Duke Math. J. 
[Han57] J.-I. Hano, On Kaehlerian homogeneous spaces of unimodular Lie groups, Amer. J. Math. 79 (1957), $885-900$

[Kik11] S. Kikuta, Carathéodory measure hyperbolicity and positivity of canonical bundles, Proc. Amer. Math. Soc. 139 (2011), no. 4, 1411-1420.

[Kik13]__ Restricted Carathéodory measure and restricted volume of the canonical bundle, Michigan Math. J. 62 (2013), no. 2, 259-292.

[Kob59] S. Kobayashi, Geometry of bounded domains, Trans. Amer. Math. Soc. 92 (1959), 267-290.

[Kob98] _ Hyperbolic complex spaces, Grundlehren der Mathematischen Wissenschaften [Fundamental Principles of Mathematical Sciences], vol. 318, Springer-Verlag, Berlin, 1998.

[Kod54] K. Kodaira, On Kähler varieties of restricted type (an intrinsic characterization of algebraic varieties), Ann. of Math. (2) 60 (1954), 28-48.

[Kol95] J. Kollár, Shafarevich maps and automorphic forms, M. B. Porter Lectures, Princeton University Press, Princeton, NJ, 1995.

[Lan86] S. Lang, Hyperbolic and Diophantine analysis, Bull. Amer. Math. Soc. (N.S.) 14 (1986), no. 2, 159-205.

[Liu96] K. Liu, Geometric height inequalities, Math. Res. Lett. 3 (1996), no. 5, 693-702.

[McQ98] M. McQuillan, Diophantine approximations and foliations, Inst. Hautes Études Sci. Publ. Math. (1998), no. 87, 121-174.

[McQ99] _ Holomorphic curves on hyperplane sections of 3-folds, Geom. Funct. Anal. 9 (1999), no. 2, 370-392.

[MM83] S. Mori and S. Mukai, The uniruledness of the moduli space of curves of genus 11, Algebraic geometry (Tokyo/Kyoto, 1982), Lecture Notes in Math., vol. 1016, Springer, Berlin, 1983, pp. 334-353.

[Nad89] A. M. Nadel, The nonexistence of certain level structures on abelian varieties over complex function fields, Ann. of Math. (2) 129 (1989), no. 1, 161-178.

[Pac04] G. Pacienza, Subvarieties of general type on a general projective hypersurface, Trans. Amer. Math. Soc. 356 (2004), no. 7, 2649-2661.

[RY18] E. Riedl and D. Yang, Applications of a grassmannian technique in hypersurfaces, preprint arXiv:1806.02364 (2018).

[Ros79] J.-P. Rosay, Sur une caractérisation de la boule parmi les domaines de $\mathrm{C}^{n}$ par son groupe d'automorphismes, Ann. Inst. Fourier (Grenoble) 29 (1979), no. 4, ix, 91-97.

[Rou16] E. Rousseau, Hyperbolicity, automorphic forms and Siegel modular varieties, Ann. Sci. Éc. Norm. Supér. (4) 49 (2016), no. 1, 249-255.

[RT18] E. Rousseau and F. Touzet, Curves in Hilbert modular varieties, Asian J. Math. 22 (2018), no. 4, 673-690.

[Sib81] N. Sibony, A class of hyperbolic manifolds, Recent developments in several complex variables (Proc. Conf., Princeton Univ., Princeton, N. J., 1979), Ann. of Math. Stud., vol. 100, Princeton Univ. Press, Princeton, N.J., 1981, pp. 357-372.

[Sie50] C. L. Siegel, Analytic Functions of Several Complex Variables, Institute for Advanced Study, Princeton, N.J., 1950, Notes by P. T. Bateman. 
[Siu04] Y.-T. Siu, Hyperbolicity in complex geometry, The legacy of Niels Henrik Abel, Springer, Berlin, 2004, pp. 543-566.

[Siu15] - Hyperbolicity of generic high-degree hypersurfaces in complex projective space, Invent. Math. 202 (2015), no. 3, 1069-1166.

[TY17] V. Tosatti and X. Yang, An extension of a theorem of Wu-Yau, J. Differential Geom. 107 (2017), no. 3, 573-579.

[Voi96] C. Voisin, On a conjecture of Clemens on rational curves on hypersurfaces, J. Differential Geom. 44 (1996), no. 1, 200-213.

[Won77] B. Wong, Characterization of the unit ball in $\mathbf{C}^{n}$ by its automorphism group, Invent. Math. 41 (1977), no. 3, 253-257.

[WY16a] D. Wu and S.-T. Yau, Negative holomorphic curvature and positive canonical bundle, Invent. Math. 204 (2016), no. 2, 595-604.

[WY16b] _ A remark on our paper "Negative holomorphic curvature and positive canonical bundle", Comm. Anal. Geom. 24 (2016), no. 4, 901-912.

[Xu94] G. Xu, Subvarieties of general hypersurfaces in projective space, J. Differential Geom. 39 (1994), no. 1, $139-172$.

[Zim19] A. Zimmer, Smoothly bounded domains covering compact manifolds, preprint arXiv:1910.05288 (2019). 